\title{
Demonstration of the Elusive Concentration-Gradient Paramagnetic Force
}

\author{
Nicholas Leventis, ${ }^{1 *}$ Amala Dass ${ }^{2}$
}

1. Materials Division, NASA Glenn Research Center, 21000 Brookpark Road. M.S. 49-1, Cleveland, OH 44135; Tel.: 216-433-3202; E-mail:

Nicholas.Leventis@nasa.gov

2. Department of Chemistry, University of Missouri-Rolla, Rolla, MO 65409; Tel.: 573-341-4033; E-Mail: Amal@umr.edu

\section{Supporting Online Material}

Appendix I: $\quad$ Experimental Section

Appendix II: Concentration profiles along a diffusion-controlled voltammogram of nitrobenzene

Appendix III: Derivation of eq 3, Data Analysis, Other Systems

1. The charge, $Q$, passing during voltammetry is inversely proportional to the square root of the potential sweep rate

2. The weight gain or loss by the diffusion layer during voltammetry depends on the potential sweep rate

3. Balance of Forces and Equation 3

4. Data analysis according to eq 3

Movies: $\quad$ S1 - S6 (in six separate .MOV files)

Legends for Movies S1 - S6 


\section{Appendix I: Experimental Section}

Materials. All chemicals were purchased from Aldrich unless noted otherwise. Nitrobenzene (NB) was distilled twice under reduced pressure before use. $N, N, N^{\prime}, N^{\prime}$ tetramethyl-p-phenylenediamine (TMPD) was sublimed. Acetonitrile, anhydrous $99.8 \%$ (Aldrich) was used as received. Supporting electrolyte tetrabutylammonium perchlorate (TBAP) was prepared from tetrabutylammonium bromide and perchloric acid as described before ${ }^{1}$. Sodium perchlorate was dried overnight under vacuum at $60{ }^{\circ} \mathrm{C}$.

Equipment. Electrochemical experimentation was conducted in Ar-degassed solutions using a Perkin-Elmer 263A potentiostat controlled by the Perkin-Elmer Model 270/250 Research Electrochemical Software 4.30. Au, Pt and Fe working electrodes were made by sealing short $1.00 \mathrm{~mm}$ diameter wires (Alfa Aesar) in bent glass tubes with white epoxy. Contact to the $\mathrm{Au}, \mathrm{Pt}$ or $\mathrm{Fe}$ electrodes was made by winding $\mathrm{Cu}$ wire around them, while silver paint was used to ensure good contact. The exposed surface was polished first with fine sand paper followed with diamond paste (successively $5 \mu \mathrm{m}, 3 \mu \mathrm{m}, 1 \mu \mathrm{m}$ ). An aqueous $\mathrm{Ag} / \mathrm{AgCl}$ reference electrode was purchased from $\mathrm{CH}$ Instruments, Inc. (Austin, TX). The counter electrode was a Pt mesh (Alfa Aesar).

A magnetic field was generated by an electromagnet constructed around two iron pole pieces, $9 \mathrm{~cm}$ in diameter extending $4.5 \mathrm{~cm}$ out of the $\mathrm{Cu}$ coils and leaving a gap of 3 $\mathrm{cm}$. The two poles were mounted with iron posts on a 2 inch thick iron base plate. The coils were powered with a Kepco Inc. (Flushing, NY) power supply, model JQE 150-1.5 with maximum DC current output of $1.5 \mathrm{~A}$. With the power supply at the maximum DC current output of $1.5 \mathrm{~A}$, the field generated in the gap between the centers of the two poles is $3.3 \mathrm{~T}$. Owing to the critical importance of being able to measure the magnetic induction accurately, the ability of our testa-meter to measure the magnetic field reliably, was tested by plotting the measured field intensity vs. the current flowing through the coils of our electromagnet. The plot was linear over the entire $0 \mathrm{~T}-3.3 \mathrm{~T}$ range with $\mathrm{R}^{2}=0.999$. The field was $3.24 \mathrm{~T}$ one inch away from the center of the poles (a $1.8 \%$ decrease). Thus, it is calculated that the magnetic field felt across the diameter $(1.00 \mathrm{~mm})$

1. Leventis, N.; Gao, X. J. Phys. Chem. B 1999, 103, 5832-5840. 
of our disk electrodes placed near the center of the gap between the poles of the electromagnet is for all practical purposes homogeneous within $0.23 \%$.

Digital Optical photography was conducted under usual laboratory lighting conditions augmented with a desk fluorescent lamp using a Nikon CoolPix 5000 digital camera placed $7 \mathrm{~cm}$ away from the electrode, using the macro mode, manual focus and the auto balance setting. Adobe Photoshop ${ }^{\mathrm{TM}}$ was used to cut the region of interest from the camera TIFF format output file and was used without further modification.

Movies of the diffusion layer (QVGA, $320 \times 240$ pixels in size) were shot at 15 frames per second for the possible maximum of 60 seconds with the same Nikon Coolpix 5000 digital camera and stored as Quicktime movie files. The camera settings were the same as that of the photographs described above. The cyclic voltammetric data corresponding to the each video were recorded and used for animation.

For animation, cyclic voltammetric data were imported into MATLAB (Version 6.0.0.88 Release 12). Animated cyclic voltammogram was obtained with the 'avifile' command as an uncompressed '.AVI' movie file (at 15 frames per second).

For the Supporting Movie files, the above videos were imported as embedded videos into Macromedia FlashMX Professional 2004 (Version 7.2). Editing was done when necessary, by selecting the region of interest and clipping off the unwanted initial, final and audio tracks of the movie. Each movie was placed in separate layers. The labeling was done with the text tool in a separate layer. This finished movie was exported as an uncompressed 'AVI' file. The screen size of the movie was chosen while exporting the '.AVI' file, and was maintained till the end to ensure good quality movies. The AVI file was imported into Quicktime Player (Version 6.5.2); MPEG-4 Video was used for compression and the resulting file was exported as '.MOV' file. 


\section{Appendix II: Concentration profiles along a diffusion-controlled voltammogram of nitrobenzene (This information is used for explaining the loss of the return wave at the platinum electrode (refer to Figure 1, red line, in the Pt electrode frame).}

Figure S1 below shows the simulated cyclic voltammogram of nitrobenzene (NB), calculated by setting $E_{1 / 2}=-1.09 \mathrm{~V}$ vs. $\mathrm{Ag} / \mathrm{AgCl}$, and by using as the diffusion coefficients of NB and its anion radical NB- ${ }^{-}$the values: $D_{\mathrm{NB}}=2.4 \times 10^{-5} \mathrm{~cm}^{2} \mathrm{~s}^{-1}{ }^{2}$ ), and $D_{\mathrm{NB} \cdot}-/ D_{\mathrm{NB}}=0.90$, measured here from the forward and return peak current ratio by cyclic voltammetry at $100 \mathrm{mVs}^{-1}$. All simulations were conducted using code in FORTRAN 77 based on well-established explicit finite difference integration methods. ${ }^{3}$

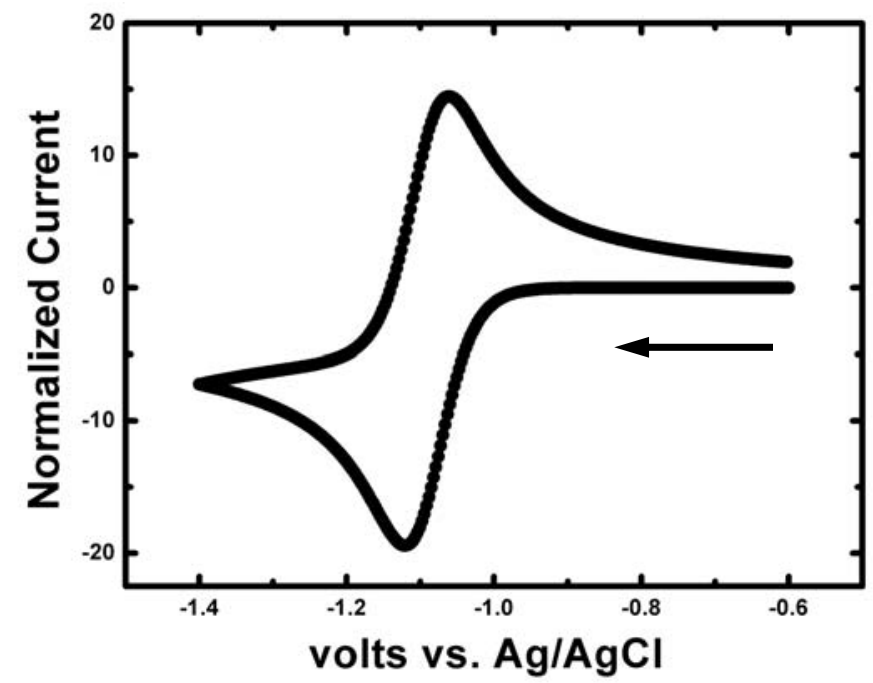

Figure S1. Simulated cyclic voltammetry of NB.

Figure S2-S4 show the concentration profiles away from the electrode of NB and of paramagnetic $\mathrm{NB}^{-}$at the forward peak-current potential $(-1.120 \mathrm{~V}$ vs. $\mathrm{Ag} / \mathrm{AgCl})$, at the return potential $(-1.400 \mathrm{~V}$ vs. $\mathrm{Ag} / \mathrm{AgCl})$ and at the return peak-current potential $(-1.060 \mathrm{~V}$ vs. $\mathrm{Ag} / \mathrm{AgCl}$ ), respectively. Note that at the return peak-current potential (Fig. S-4), the concentration of $\mathrm{NB}^{-}$reaches maximum not at, but near the electrode. The $\mathrm{NB}^{-}$

2. Malmsten, R. A.; Smith, C. P.; White, H. S. J. Electroanal. Chem. 1986, 215, 223 235.

3. (a) Leventis, N.; Gao, X. J. Phys. Chem. B 1999, 103, 5832-5840.

(b) Collins, C. M.; Sotiriou-Leventis, C.; Canalas, M. T.; Leventis, N. Electrochimica Acta 2000, 45, 2049-2059.

(c) Leventis, N.; Gao, X. J. Electroanal. Chem. 2001, 500, 78-94.

(d) Leventis, N.; Oh, W. S.; Gao, X.; Rawashdeh, A.-M., M. Anal. Chem. 2003, 75, 4996-5005. 
concentration decreases more sharply towards the electrode and more smoothly towards the bulk. In other words, considering the concentration gradient from the point of maximum $\left[\mathrm{NB}^{-}\right]$, we realize that $\mid \nabla C_{\rightarrow}$ electrode $|>| \nabla C_{\rightarrow \text { bulk }} \mid$, and therefore of the two possible $\boldsymbol{F}_{\nabla \mathrm{VC}}$ forces, the $\boldsymbol{F}_{\mathrm{\nabla C}}$ force that tries to retain the diffusion layer is weaker than the $\boldsymbol{F}_{\mathrm{VC}}$ force that tries to remove it (see also legend of Fig. S4). The result is that the diffusion layer is removed and the return wave is lost (refer to: (a) the red line curve in Figure 1 - data with Pt electrode facing the field, $\theta=0^{\circ}$; and, (b) movies S1-S3).

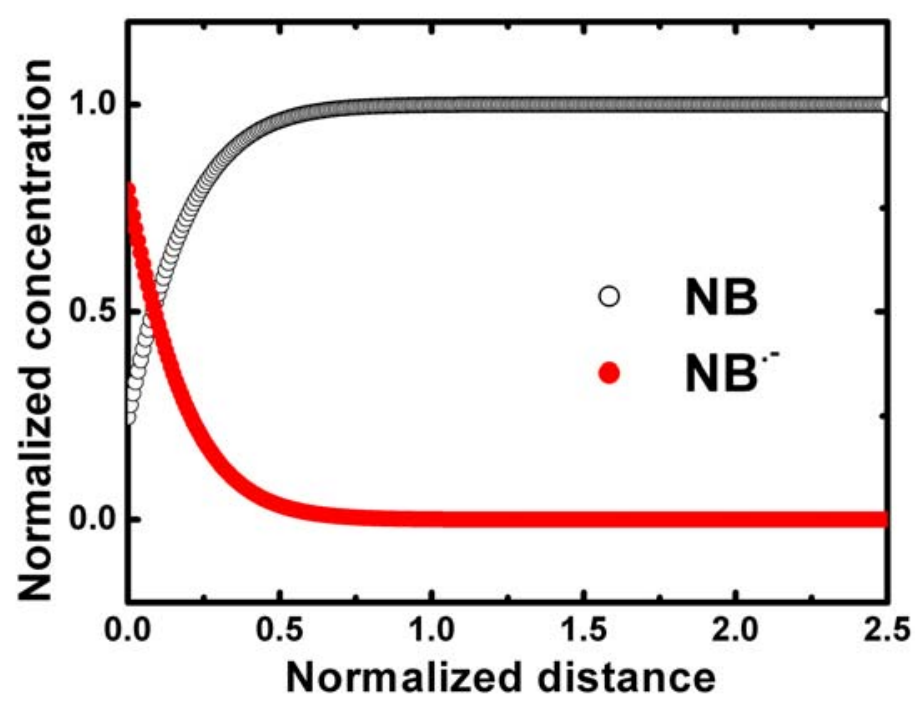

Figure S2. Concentration profiles at the forward peak current potential (at $-1.120 \mathrm{~V}$ vs. $\mathrm{Ag} / \mathrm{AgCl})$. 


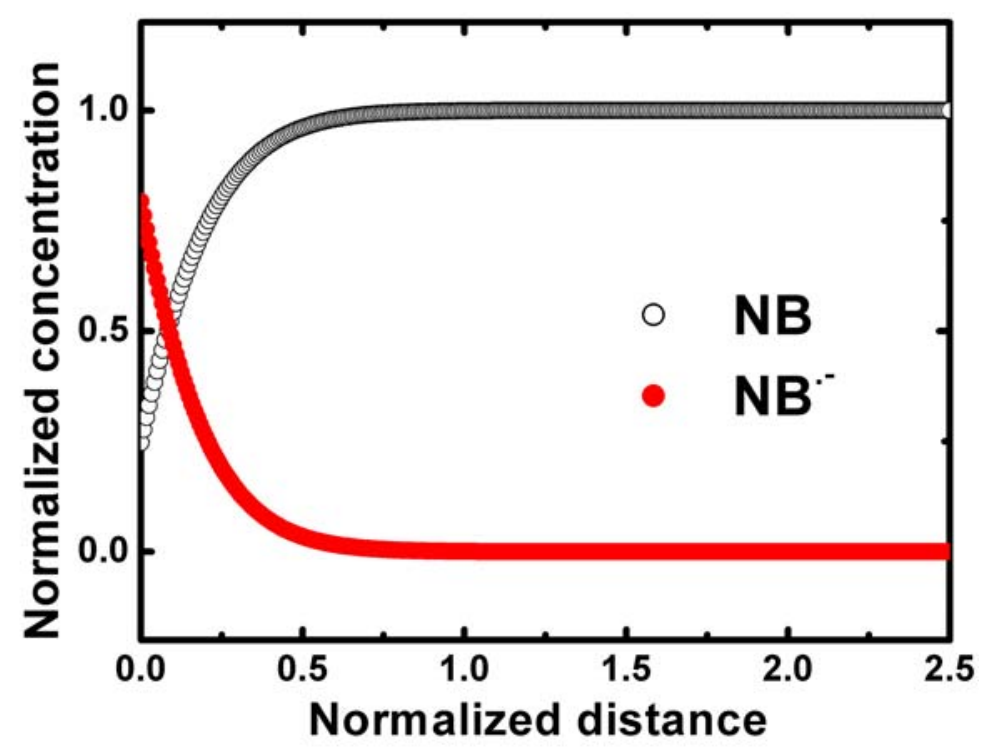

Figure S3. Concentration profiles at the return potential (at $-1.400 \mathrm{~V}$ vs. $\mathrm{Ag} / \mathrm{AgCl}$ ).

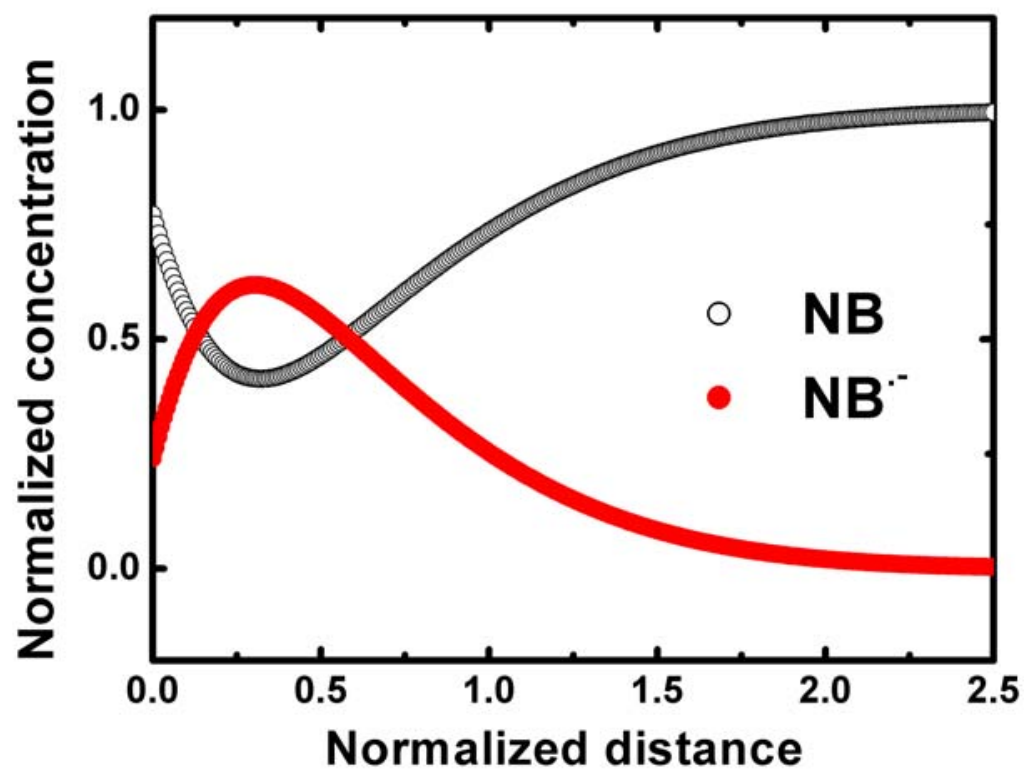

Figure S4. Concentration profiles at the return peak current potential $(-1.060 \mathrm{~V}$ vs. $\mathrm{Ag} / \mathrm{AgCl})$. Note that the concentration profile of paramagnetic $\mathrm{NB}^{-}$is asymmetric and decays faster towards the electrode and slower towards the bulk. Thus, of the two $\boldsymbol{F}_{\nabla \mathrm{C}}$ forces, (which are both directed towards the point of maximum concentration), the $\boldsymbol{F}_{\nabla \mathrm{C}}$ that tries to remove the diffusion layer towards the bulk is stronger than the $\boldsymbol{F}_{\nabla \mathrm{C}}$ that tries to retain it. 


\section{Appendix III: Derivation of eq 3, Data Analysis, Other Systems}

\section{Eq 3:}

$$
\text { weight supported magnetically } \begin{aligned}
(W) & =\left[\left(Q_{\mathrm{B}}-Q_{|\mathrm{B}|=0}\right) / n F\right]\left[F W_{+} t_{+}-F W_{-} t_{-}\right]|g|= \\
& =<\left|F_{\nabla C}\right|>V \cos \phi
\end{aligned}
$$

1. The charge, $Q$, passing during voltammetry is inversely proportional to the square root of the potential sweep rate.

Without loss of generality, under Nernstian conditions a linear sweep voltammogram (i.e., a current vs. potential curve) is given by eq S.1: ${ }^{4}$

$$
i(\sigma)=n F A C_{O}^{*}\left(\pi D_{O} \sigma\right)^{1 / 2} \chi(\sigma)
$$

where $\sigma=\frac{n F}{R T} v$, and therefore $\sigma$ is a dimensionless quantity, which, however, can be also interpreted as "potential" in units of $\frac{R T}{n F}$, so eq S.1 is essentially a function of the type $i(E)=f(E)$. (Other symbols: $n$ is the number of electrons, $F$ Faraday's Constant, $A$ the electrode area, $C_{O}^{*}$ and $D_{O}$ the bulk concentration and diffusion coefficient of species "O," and $\chi(\sigma t)$ is the normalized current function, common to all linear diffusioncontrolled linear sweep voltammograms.)

Now, in linear sweep voltammetry $E(t)=E_{\text {initial }}-v t \Rightarrow \frac{d E}{d t}=-v \Rightarrow d t=\frac{d E}{-v}$

And therefore, as far as charge, $Q$, is concerned:

$$
d Q=i(E) d t=i(E) \frac{d E}{-v}
$$

(we write $\mathrm{i}(E)$ rather than $i(\mathrm{t})$ because in a voltammogram $i$ is given as a function of $E$, not of $t$.)

So, the charge under the voltammogram is given by:

4. Bard, A. J.; Faulkner, L. R. Electrochemical Methods, Fundamentals and Applications, $2^{\text {nd }}$ ed. John Wiley and Sons: New York, 2000; page 230. 


$$
Q=\int_{E_{\text {initial }}}^{E_{\text {fipal }}} i(E) \frac{d E}{-v}=n F A C_{O}^{*} \pi^{1 / 2} D_{O}^{1 / 2}\left(\frac{n F}{R T}\right)^{1 / 2} \frac{1}{-v^{1 / 2}} \int_{E_{\text {initial }}}^{E_{\text {fipal }}} \chi(E) d E
$$

The quantity $\int_{E_{\text {initial }}}^{E_{\text {fipal }}} \chi(E) d E$ is common to all linear sweep voltammograms controlled by linear diffusion, and therefore we see from eq S.2 that:

$$
Q=\operatorname{const} \frac{1}{v^{1 / 2}}
$$

where: const $=-n F A C_{O}^{*} \pi^{1 / 2} D_{O}^{1 / 2}\left(\frac{n F}{R T}\right)^{1 / 2} \int_{E_{\text {initital }}}^{E_{\text {fipal }}} \chi(E) d E$

Hence, from eq S.3 we see that the lower the potential sweep rate, the higher the charge passing through the electrode. And, since the amount of material been electrolyzed is given by $\frac{|Q|}{n F}$, therefore:

$$
\text { moles electrolyzed }=A C_{O}^{*} \pi^{1 / 2} D_{O}^{1 / 2}\left(\frac{n F}{R T}\right)^{1 / 2} \frac{1}{v^{1 / 2}} \int_{E_{\text {intitial }}}^{E_{\text {fpal }}} \chi(E) d E
$$

i.e., the diffusion layer contains more electrolysis product the lower the potential sweep rate is.

2. The weight gain or loss by the diffusion layer during voltammetry depends on the potential sweep rate.

In the meanwhile, the charge injected in the diffusion layer during electrolysis (see eqs S.2 and S.3) is compensated by counter ions of the supporting electrolyte moving in and out the diffusion layer according to their relative transport numbers, $t_{+}$and $t_{\text {- }}$ for cations and anions, respectively. The weight change of the diffusion layer that is associated with the movement of counter ions is calculated via Newton's second law and is given by eq S.5:

weight change of the diffusion layer $=($ moles electrolyzed $) \times\left[F W_{+} t_{+}-F W_{-} t_{-}\right]|\boldsymbol{g}|$ 
where $F W_{+}$and $F W_{-}$are the formula weights of the cation and anion of the supporting electrolyte and $\boldsymbol{g}$ is the acceleration of gravity. ${ }^{5}$

\section{Balance of Forces and Equation 3}

By imposing a magnetic field at $\theta=0^{\circ}$ relative to the surface of the disk electrode, the breakpoint of the linear Randles-Sevcik relationship between the voltammetric peak current and the square root of the sweep rate moves to lower sweep rates, meaning, according to eqs S.4 and S.5, that the magnetic force can support an extra weight. Clearly then, at the respective breakpoints the extra weight is counterbalanced exactly by the magnetic force. That extra weight (i.e., the weight supported magnetically, $W$ ) is given by

weight supported magnetically $(W)=\left[\left(Q_{\mathrm{B}}-Q_{|\mathrm{B}|=0}\right) / n F\right]\left[F W_{+} t_{+}-F W_{-} t_{-}\right]|\boldsymbol{g}|$

and therefore eq 3 :

$$
W=\left[\left(\mathrm{Q}_{\mathrm{B}}-\mathrm{Q}_{|\mathrm{B}|=0}\right) / n F\right]\left[F W_{+} t_{+}-F W_{-} t_{-}\right]|g|=\left\langle\left|\boldsymbol{F}_{\nabla \mathrm{C}}\right|>V \cos \phi,\right.
$$

is obtained by realizing that the numerical value of the extra weight supported magnetically must be equal to the projection of the average magnetic force in the vertical direction, $\left\langle\left|\boldsymbol{F}_{\nabla \mathrm{C}}\right|>V \cos \phi\right.$, where $\left\langle\left|\boldsymbol{F}_{\nabla \mathrm{C}}\right|>\right.$ is the average value of $\boldsymbol{F}_{\nabla \mathrm{C}}$ per unit volume, $V$ is the volume of the diffusion layer, and $\phi$ is the angle between $\left\langle\boldsymbol{F}_{\nabla C}>V\right.$ and the vertical direction. Schemes S1 and S2 present a qualitative analysis of the body forces on the diffusion layer. A quantitative estimation of the angle $\phi$ is given below.

Scheme S1. Qualitative analysis of the forces in the magnetic field on diffusion layers that tend to rise (e.g., nitrobenzene (NB), N,N'-diheptylviologen (DHV)) ${ }^{*}$

5. Leventis, N.; Gao, X. J. Am. Chem. Soc. 2002, 124, 1079-1088. 


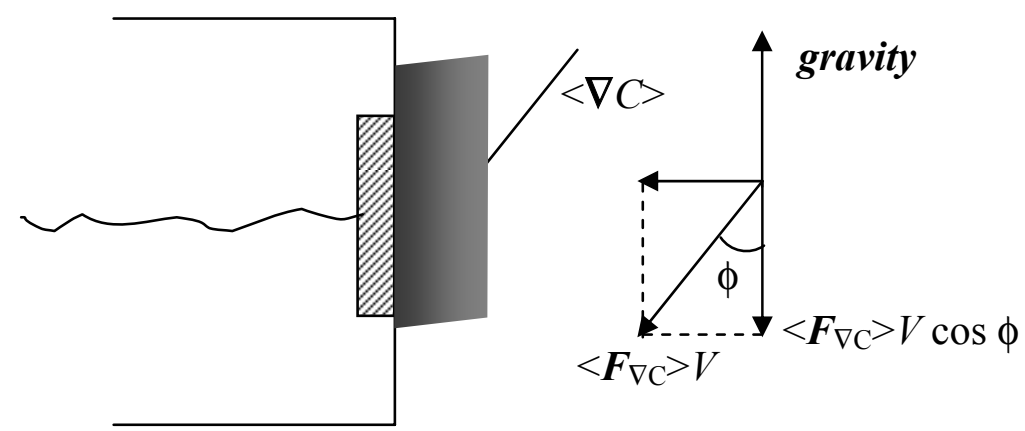

${ }^{*}$ The diffusion layer is skewed upwards until the vertical component of $\left\langle\boldsymbol{F}_{\nabla \mathrm{C}}>V\right.$ counterbalances the gravitational force that causes the layer to rise when $|\boldsymbol{B}|=0 .(<\nabla C>$ is the average concentration gradient over the diffusion layer.)

Scheme S2. Qualitative analysis of the forces in the magnetic field on diffusion layers that tend to fall (e.g., $N, N, N^{\prime}, N^{\prime}$-tetramethyl- $p$-phenylene diamine: TMPD) ${ }^{* *}$

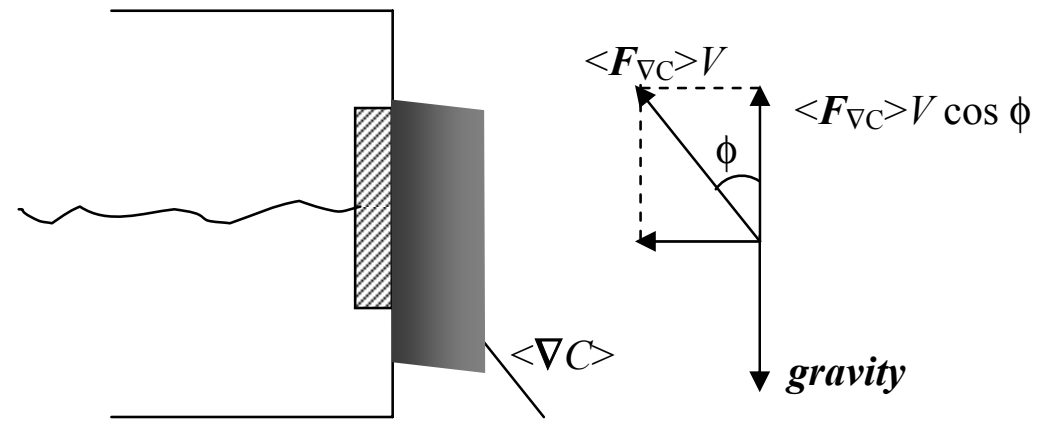

${ }^{* *}$ The diffusion layer is skewed downwards until the vertical component of $\left\langle\boldsymbol{F}_{\nabla \mathrm{C}}>V\right.$ counterbalances the gravitational force that causes the layer to fall when $|\boldsymbol{B}|=0 .(\langle\nabla C\rangle$ is the average concentration gradient over the diffusion layer.)

In reality, the skewing of the diffusion layer depicted in Schemes S1 and S2 should be very small as estimated by looking at Fig. 2B in the paper or at the photographs of Figure S5 and S6, concerning reduction of $N, N^{\prime}$-diheptylviologen (DHV, see Fig. S5) and oxidation of $N, N, N^{\prime}, N^{\prime}$-tetramethyl-p-phenylene diamine (TMPD, see Fig. S6), respectively. As expected from a $\boldsymbol{F}_{\nabla \mathrm{C}}$ perspective, the diffusion layers containing blue paramagnetic $\mathrm{DHV}^{+}$or $\mathrm{TMPD}^{+}$are retained. 

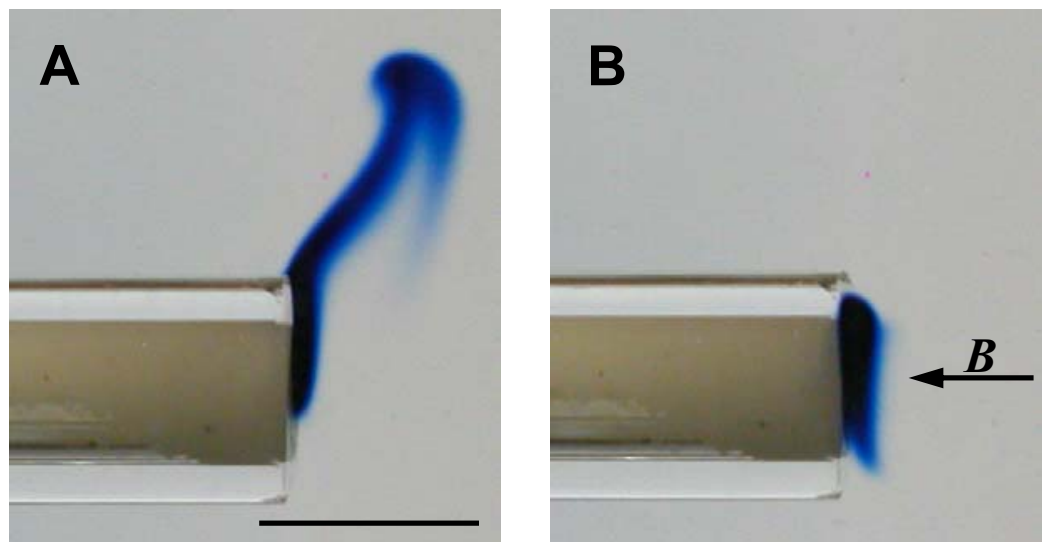

Figure S5. Photographs of a Au electrode in a $0.05 \mathrm{M}$ solution of diamagnetic DHV in $\mathrm{CH}_{3} \mathrm{CN} / 0.5 \mathrm{M}$ TBAP, $30 \mathrm{~s}$ after a potential step in the one-electron mass transfer controlled reduction range. (A) $|\boldsymbol{B}|=0$. (B) $|\boldsymbol{B}|=3.3 \mathrm{~T}, \theta=0^{\circ}$. ( $E_{\text {initial }}=-0.100 \mathrm{~V}$ vs. $\mathrm{Ag} / \mathrm{AgCl} ; E_{\text {final }}=-0.600 \mathrm{~V}$ vs. $\left.\mathrm{Ag} / \mathrm{AgCl}\right)$. The line at the bottom of frame $\mathrm{A}$ is $3.1 \mathrm{~mm}$.
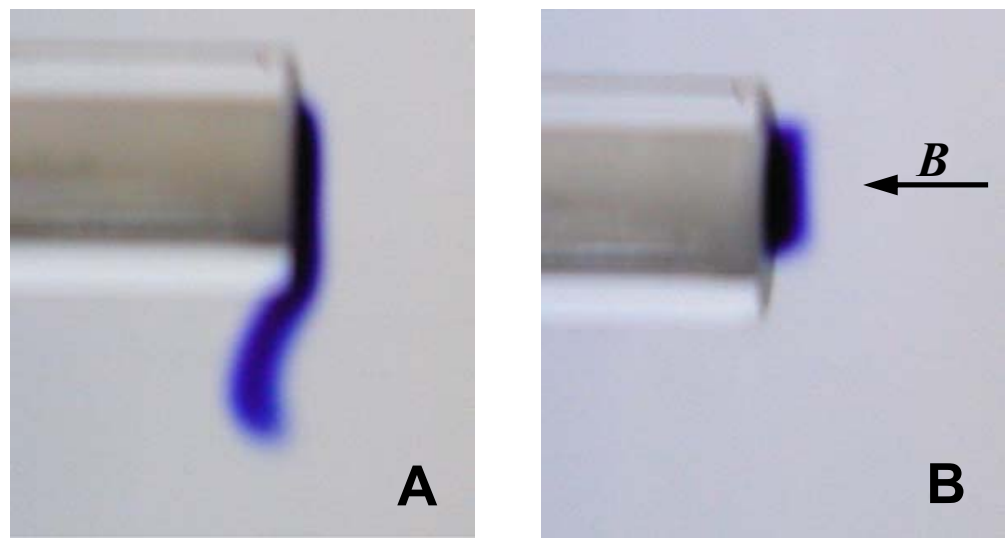

Figure S6. Photographs of a $\mathrm{Au}$ electrode in a $0.05 \mathrm{M}$ solution of diamagnetic TMPD in $\mathrm{CH}_{3} \mathrm{CN} / 0.5 \mathrm{M} \mathrm{NaClO}$, $15 \mathrm{~s}$ after a potential step in the one-electron mass transfer controlled oxidation range. (A) $|\boldsymbol{B}|=0$. (B) $|\boldsymbol{B}|=3.3 \mathrm{~T}, \theta=0^{\circ}$. ( $E_{\text {initial }}=-0.200 \mathrm{~V}$ vs. $\mathrm{Ag} / \mathrm{AgCl} ; E_{\text {final }}=0.300 \mathrm{~V}$ vs. $\left.\mathrm{Ag} / \mathrm{AgCl}\right)$.

Quantitatively, the skewing of the diffusion layer, $\phi$, can be calculated by the experimentally determined gravitational force that is counterbalanced magnetically, $W$, 
and the value of the concentration gradient paramagnetic force that is exerted upon the entire diffusion layer $\left(<\left|\boldsymbol{F}_{\nabla \mathrm{C}}\right|>V\right)$. That is from eq 3 we obtain:

$$
\cos \phi=\mathrm{W} /<\left|\boldsymbol{F}_{\nabla C}\right|>V
$$

The volume $V$ of the diffusion layer is given approximately by $V=A \delta$, where $A$ is the electrode area and $\delta$ is the diffusion layer thickness. Thus, taking into consideration eq 2 in the paper, we obtain:

$$
<\left|\boldsymbol{F}_{\nabla \mathrm{C}}\right|>V=N_{\mathrm{A}}\left[\left(g \mu_{\mathrm{B}}\right)^{2} / 4 k T\right]|\boldsymbol{B}|^{2}<\left|\nabla C_{\mathrm{NB} \cdot-}\right|>A \delta
$$

The average concentration gradient across the diffusion layer can be approximated by:

$$
<\left|\nabla C_{\mathrm{NB} \cdot-}\right|>=\frac{\Delta C_{\mathrm{NB}^{-}, \text {across the diffusion laye }} \quad r}{\delta}
$$

Therefore, eq S.8 becomes:

$$
<\left|\boldsymbol{F}_{\nabla \mathrm{C}}\right|>V=N_{\mathrm{A}}\left[\left(g \mu_{\mathrm{B}}\right)^{2} / 4 k T\right]|\boldsymbol{B}|^{2} \Delta C_{\mathrm{NB}^{-}, \text {across the diffusion layer }} \quad A
$$

(Note that the diffusion layer thickness (a source of uncertainty) has been cancelled out in eq S.9.)

The values and units of the various quantities in eq S.9 are as follows:

$$
\begin{aligned}
& N_{\mathrm{A}}=6.023 \times 10^{23} \mathrm{~mol}^{-1} \\
& g=2 \\
& \mu_{\mathrm{B}}=9.274 \times 10^{-24} \mathrm{~J} \mathrm{~T}^{-1} \\
& k=1.38 \times 10^{-23} \mathrm{~J} \mathrm{~K}^{-1} \\
& T=298 \mathrm{~K} \\
& \Delta C_{\mathrm{NB}^{-}}, \text {across the diffusion layer }=0.1 \mathrm{M}=10^{2} \mathrm{~mol} \mathrm{~m}^{-3} \\
& \mathrm{~A}=\pi \times(\text { electrode radius })^{2}=\pi \times\left(0.5 \times 10^{-3}\right)^{2} \mathrm{~m}
\end{aligned}
$$

For the value of $|\boldsymbol{B}|$, let us choose, for example, $|\boldsymbol{B}|=2 \mathrm{~T}$. Thus, from eq S.9 we calculate that $<\left|\boldsymbol{F}_{\nabla \mathrm{C}}\right|>V=3.95 \times 10^{-6} \mathrm{~N}$.

Meanwhile, from Fig. 3B in the paper we see that at $|\boldsymbol{B}|=2 \mathrm{~T}$, the experimentally found weight that is supported magnetically is $W=10^{-8.4} \mathrm{~N}$. Therefore, $\cos \phi=10^{-8.4} / 3.95 \times 10^{-}$ ${ }^{6}=0.001$, hence $\phi=89.9^{\circ}$, meaning that $\boldsymbol{F}_{\nabla \mathrm{C}}$ is still directed almost perpendicular to the electrode. This analysis explains the apparent paradox where a vertical force (gravity) is apparently counterbalanced by a force that should (and appears to) be directed 
horizontally. Simply, the concentration gradient paramagnetic force is much stronger than gravity, as we have discussed before. ${ }^{5}$

Now, the horizontal component of the magnetic force, $\left\langle\boldsymbol{F}_{\mathrm{VC}}>V \sin \phi\right.$, is counteracted by the electrode. But, if the susceptibility gradient were free to move, it would be propelled. This is what is observed with permanent susceptibility gradients generated by aligning iron particles in silica aerogels monoliths as those shown in Fig. S7.

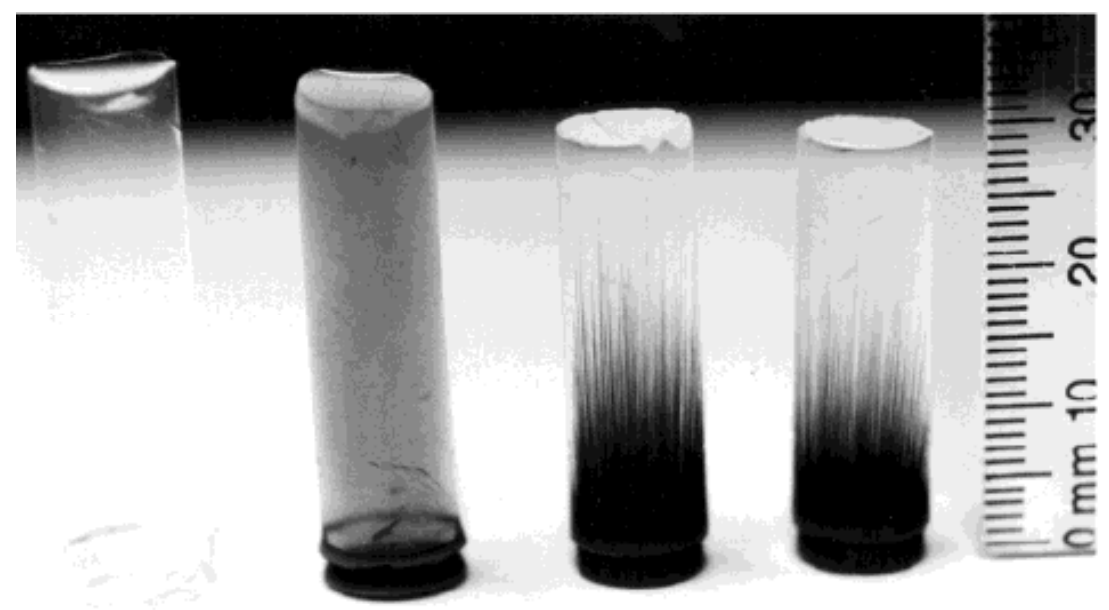

Figure S7. Silica aerogel monoliths with permanent susceptibility gradients (two samples on the right). For comparison: plain silica aerogel monolith (left) and with a random distribution of iron particles (second sample from the left). Taken from: Leventis, N.; Elder, I. A.; Long, G. J.; Rolison, D. R. Nano Lett. 2002, 2, 63-67.

\section{Data Analysis According to eq 3.}

Typical linear sweep voltammograms (LSVs) at $|\boldsymbol{B}|=0$ and variable sweep rates are shown in the left frame of Figure S8. The peak current is identified as the highest absolute current in each voltammogram. When no longer an actual peak current can be detected, the limiting current becomes the highest absolute current, and the mass transfer is dominated by natural convection. When a magnetic field is imposed, similar families of LSV-waves are obtained at lower sweep rates (see right frame in Figure S8). (In this context, also consider the data shown in Figure 1 of the paper, where at $10 \mathrm{mVs}^{-1}$ and $|\boldsymbol{B}|=0$ mass transfer is dominated by natural convection, but when $\boldsymbol{B}$ is "on" we see a regular diffusion wave.) Thus, from similar families of LSV-curves at various $|\boldsymbol{B}|$ 's we 
obtain curves like those as shown in Figure S9 (and included in the main body of the paper as Figure 3A.).

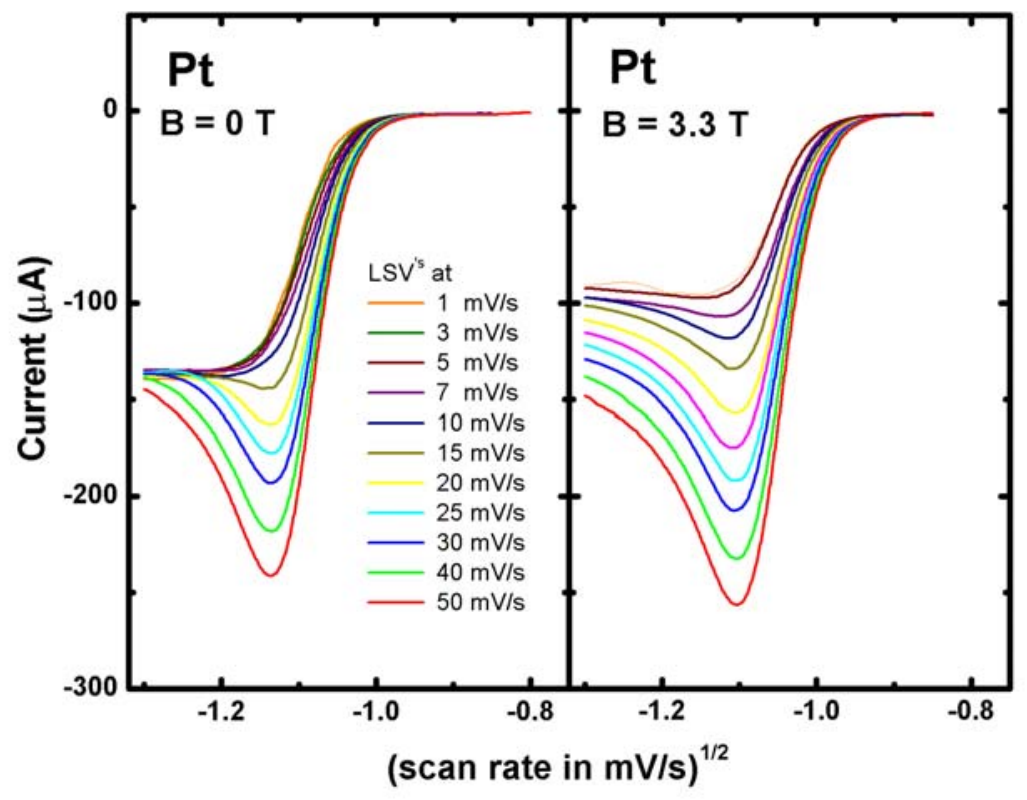

Figure S8. Typical data for finding the diffusion layer weight supported by $\boldsymbol{F}_{\nabla \mathrm{C}}$ at various $|\boldsymbol{B}|$ s. Left: linear sweep voltammograms in a $0.1 \mathrm{M} \mathrm{NB}$ solution in $\mathrm{CH}_{3} \mathrm{CN} / 0.5 \mathrm{M}$ TBAP at variable sweep rates in the absence of a magnetic field using a Au disk electrode ( $1 \mathrm{~mm}$ in diameter). Right: Same experiment where the electrode faces $\left(\theta=0^{\circ}\right)$ a magnetic field of $|\boldsymbol{B}|=3.3 \mathrm{~T}$.

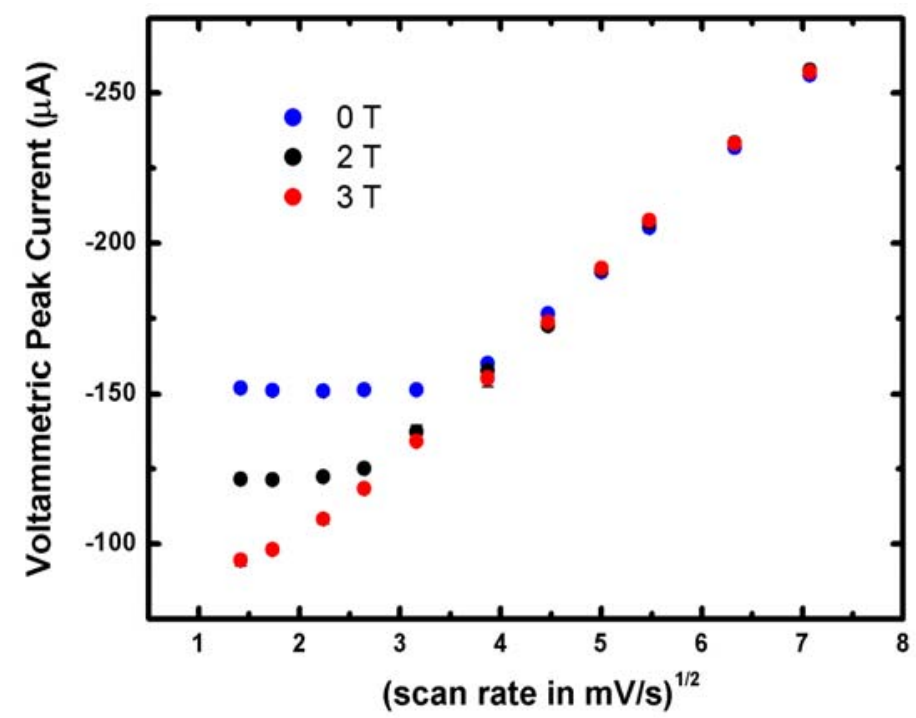

Figure S9. Randles-Sevcik plots at $\theta=0^{\circ}$ and various $|\boldsymbol{B}|$ 's, at the same sweep rates shown in Figure S8 part A. 
From curves like those of Figure S9 for each $|\boldsymbol{B}|$, we identify the sweep rate at which the linear Randles-Sevcik relationship fails, indicating the onset of natural convection. At that sweep rate, the charge, $Q$, passing through the electrode is obtained by integrating the last voltammogram recorded just before the breakpoint in the Randles-Sevcik plot. The integrals of the voltammograms were taken consistently from $E_{\text {initial }}(-0.7 \mathrm{~V}$ vs. $\mathrm{Ag} / \mathrm{AgCl})$ up to $-1.20 \mathrm{~V}$ vs. $\mathrm{Ag} / \mathrm{AgCl}$, that is just past the forward peak current at -1.13 $\mathrm{V}$ vs. $\mathrm{Ag} / \mathrm{AgCl}$. The integral charge was calculated either directly by the electrochemical software, or independently by exporting the voltammograms into Origin ${ }^{\mathrm{TM}}$ and dividing the integral area underneath the $i(E)$ curve by the sweep rate $v$, because $d t=\frac{d E}{-v}$. (As expected, the two methods gave identical results.) Subsequently, the excess weight supported by the magnetic force was calculated directly via eq S.6, where the transport numbers of the cations (tetrabutylammonium) and the anions (perchlorate) (0.37 and 0.63 , respectively) were calculated from their corresponding limiting molar ionic conductivities of 61.4 and 103.7 respectively, which were taken from the literature. ${ }^{7}$ A plot of the weight supported magnetically is linear vs. $|\boldsymbol{B}|^{2}$ with correlation $\mathrm{R}^{2}=0.994$ (Figure S10).

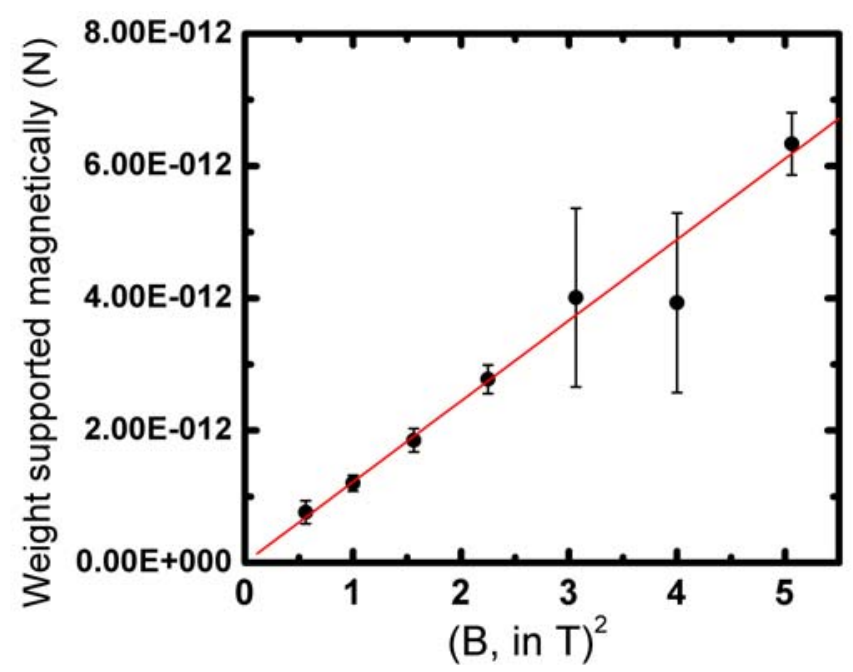

Figure S10. Plot of the weight supported magnetically versus $|\boldsymbol{B}|^{2} . \mathrm{R}^{2}=0.994$. Error bars: one standard deviation.

7. Springer, C. H.; Coetzee, J. F.; Kay, R. L. J. Phys. Chem. 1969, 73, 471-476. 


\section{Movies S1 - S6}

Six movies are attached as separate files. Although the movies are self explanatory, a list of appropriate legends is given below as an index.

Legends for Movies S1-S6

\section{Movie S1 (Quick Time - 6.4 MB)}

Synchronized video of: (a) the diffusion layer evolution at a Au disk electrode (1 mm diameter) during cyclic voltammetry; and, (b) the corresponding current-voltage curves of a $0.1 \mathrm{M}$ nitrobenzene (NB) solution in $\mathrm{CH}_{3} \mathrm{CN} / 0.5 \mathrm{M}$ TBAP at $10 \mathrm{mV} / \mathrm{s}$ at $|\boldsymbol{B}|=0 \mathrm{~T}$ and at $|\boldsymbol{B}|=3.3$ T at $\theta=0^{\circ}$.

\section{Movie S2 (Quick Time - 5.2 MB)}

Synchronized video of: (a) the diffusion layer evolution at a Au disk electrode (1 $\mathrm{mm}$ diameter) during cyclic voltammetry; (b) the corresponding concentration profiles; and, (c) the corresponding current-voltage curves of a $0.1 \mathrm{M}$ nitrobenzene (NB) solution in $\mathrm{CH}_{3} \mathrm{CN} / 0.5 \mathrm{M}$ TBAP at $10 \mathrm{mV} / \mathrm{s}$ at $|\boldsymbol{B}|=3.3 \mathrm{~T}$ at $\theta=0^{\circ}$.

\section{Movie S3 (Quick Time - 6.4 MB)}

Synchronized video of the diffusion layer evolution at a Au and a Fe disk electrode (both $1 \mathrm{~mm}$ diameter) during cyclic voltammetry in a $0.1 \mathrm{M}$ nitrobenzene (NB) solution in $\mathrm{CH}_{3} \mathrm{CN} / 0.5 \mathrm{M}$ TBAP at $10 \mathrm{mV} / \mathrm{s}$ at $|\boldsymbol{B}|=0 \mathrm{~T}$ and at $|\boldsymbol{B}|=3.3 \mathrm{~T}$

\section{Movie S4 (Quick Time - 6.4 MB)}

A $60 \mathrm{sec}$ video during potential step chronoamperometry $\left(E_{\text {initial }}=-0.925 \mathrm{~V} ; E_{\text {final }}=\right.$ $1.225 \mathrm{~V}$ vs. $\mathrm{Ag} / \mathrm{AgCl})$ with a $\mathrm{Au}$ disk electrode ( $1 \mathrm{~mm}$ diameter) in a $0.1 \mathrm{M}$ nitrobenzene (NB) solution in $\mathrm{CH}_{3} \mathrm{CN} / 0.5 \mathrm{M}$ TBAP at $|\boldsymbol{B}|=0 \mathrm{~T}$ and at $|\boldsymbol{B}|=3.3 \mathrm{~T}$. 


\section{Movie S5 (Quick Time - 3.1 MB)}

A $30 \mathrm{sec}$ video during potential step chronoamperometry $\left(E_{\text {initial }}=-0.100 \mathrm{~V} ; E_{\text {final }}=\right.$ $0.600 \mathrm{~V}$ vs. $\mathrm{Ag} / \mathrm{AgCl})$ with a $\mathrm{Au}$ disk electrode (1mm diameter) in a $50 \mathrm{mM} N, N^{\prime}-$ diheptylviologen (DHV) solution in $\mathrm{CH}_{3} \mathrm{CN} / 0.5 \mathrm{M}$ TBAP at $|\boldsymbol{B}|=0 \mathrm{~T}$ and at $|\boldsymbol{B}|=3.3 \mathrm{~T}$.

\section{Movie S6 (Quick Time - 1.5 MB)}

A $15 \mathrm{sec}$ video during potential step chronoamperometry $\left(E_{\text {initial }}=-0.200 \mathrm{~V} ; E_{\text {final }}=\right.$ $+0.300 \mathrm{~V}$ vs. $\mathrm{Ag} / \mathrm{AgCl})$ with a $\mathrm{Au}$ disk electrode ( $1 \mathrm{~mm}$ diameter) in a $50 \mathrm{mM} N, N, N^{\prime}, N^{\prime}-$ tetramethyl-p-phenylenediamine (TMPD) solution in $\mathrm{CH}_{3} \mathrm{CN} / 0.5 \mathrm{M} \mathrm{NaClO}_{4}$ at $|\boldsymbol{B}|=0 \mathrm{~T}$ and at $|\boldsymbol{B}|=3.3 \mathrm{~T}$. 